\title{
Interleukin-15 is associated with disease severity in viral bronchiolitis
}

\author{
T. Ronan Leahy, ${ }^{1,2}$, Ross McManus ${ }^{2}$, Derek G. Doherty ${ }^{3}$, Robert Grealy ${ }^{2,4}$, \\ Tanya Coulter ${ }^{3}$, Paul Smyth ${ }^{5}$, Gordon Blackshields ${ }^{5}$, Orla Sheils ${ }^{5}$, \\ Michael J. Carr ${ }^{6}$, Nikhil Purandare ${ }^{7}$, Michael Geary ${ }^{7}$, Hennie M. Hodemaekers ${ }^{8}$, \\ Riny Janssen ${ }^{8}$, Louis Bont ${ }^{8}$, Dubhfeasa Slattery ${ }^{1}$ and Thomas Ryan ${ }^{4}$
}

Affiliations: ${ }^{1}$ Dept of Paediatrics, Children's University Hospital, Dublin, Ireland. ${ }^{2}$ Dept of Clinical Medicine, Trinity College, Dublin, Ireland. ${ }^{3}$ Dept of Immunology, Trinity College, Dublin, Ireland. ${ }^{4}$ Dept of Critical Care Medicine, St James' Hospital, Dublin, Ireland. ${ }^{5}$ Dept of Histopathology, Sir Patrick Dun Research Laboratory, Trinity College, Dublin, Ireland. ${ }^{6}$ National Virus Reference Laboratory, University College Dublin, Dublin, Ireland. ${ }^{7}$ Dept of Obstetrics and Gynaecology, Rotunda Hospital, Dublin, Ireland. ${ }^{8}$ University Medical Center, Wilhelmina Children's Hospital, Utrecht, The Netherlands.

Correspondence: T. Ronan Leahy, Children's University Hospital, Temple Street, Dublin 1, Ireland.

E-mail: ronan.leahydolchc.ie

ABSTRACT Disease severity in viral bronchiolitis in infancy is difficult to predict and has been linked to host innate immunity. The study aimed to investigate the innate cytokine interleukin-15 (IL-15) as a marker of disease severity.

A prospective single-centre observational study was conducted in a university-affiliated paediatric teaching hospital, comparing children (0-18 months) hospitalised for viral bronchiolitis, those admitted to the paediatric intensive care unit with severe disease and healthy age-matched controls. IL-15-related parameters were compared between groups. PCR and microRNA (miRNA) sequencing was undertaken on natural killer (NK) cells collected from study participants.

Samples from 88 children with viral bronchiolitis and 43 controls enrolled between 2009 and 2012 were analysed. Peripheral blood mononuclear cell (PBMC) IL-15 mRNA expression was significantly higher in those with moderate severity bronchiolitis compared with controls and those with severe disease. Serum IL-15 levels correlated with disease severity. The relative frequency of NK cells in peripheral blood was significantly reduced in participants with bronchiolitis. The NK cell miRNA transcriptome in bronchiolitis was distinct. Targets of de-regulated miRNA were differentially expressed in bronchiolitis, including JAK3, STAT5A and NFKB1 on the IL-15 signalling pathway.

IL-15 is associated with disease severity in children hospitalised with viral bronchiolitis.

Interleukin-15 influences the host innate response and is associated with disease severity in viral bronchiolitis http://ow.ly/S5JfP

\footnotetext{
This article has supplementary material available from erj.ersjournals.com

Received: April 232015 | Accepted after revision: July 292015 | First published online: Nov 052015

Support statement: This study was funded by the Children's Fund for Health, Children's University Hospital, Dublin (PAC0934) and the Health Research Board (HPF/2011/017). Funding information for this article has been deposited with FundRef.
}

Conflict of interest: Disclosures can be found alongside the online version of this article at erj.ersjournals.com

Copyright CERS 2016 


\section{Introduction}

Viral bronchiolitis is the commonest single reason for an infant to be hospitalised in the developed world and the leading cause of lower respiratory tract infection in children worldwide [1]. It is commonest in children under 2 years and caused most frequently by respiratory syncytial virus (RSV) [2]. The disease spectrum ranges from asymptomatic infection to severe disease with respiratory failure requiring paediatric intensive care unit (PICU) admission for ventilator support [2,3]. Some children have predisposing risk factors, but the majority of those who require PICU admission were previously healthy $[4,5]$. Identifying children who are likely to develop severe disease is difficult and is further hampered by a lack of reliable laboratory predictors of disease.

Evidence from both functional and genetic associations studies suggest that disease severity in viral bronchiolitis is influenced by the host's innate immune response [6,7]. Natural killer (NK) cells are innate lymphocytes and are "first responders" against infection [8]. This capacity to respond quickly underlines their importance in acute respiratory infection [9-11]. NK cells also shape the subsequent adaptive immune response $[12,13]$ and failure of this response may underlie severe disease, as suggested by autopsy studies on cases of fatal viral bronchiolitis [14].

Lymphocyte homeostasis is regulated by a family of interleukins (ILs) that share a common $\gamma$-chain receptor $\left(\gamma_{c}\right)$ and mediate their effect by increasing expression of antiapoptotic proteins, including Bcl-2 and $\mathrm{Bcl}-\mathrm{x}_{\mathrm{L}}$ [15]. IL-15, in particular, regulates NK cell development and activation state [16, 17], and has previously been linked with the risk of hospitalisation for viral bronchiolitis $[6,18]$. Preliminary work examining a panel of innate cytokines in viral bronchiolitis also demonstrated a relationship between IL-15 levels and disease [19].

We hypothesised that IL-15 was a marker of disease severity in viral bronchiolitis, that genetic variation might affect the host IL-15 pathway and that IL-15 influences disease severity through its effect on NK cells.

\section{Methods}

Study participants

We conducted a single-centre, prospective observational study during consecutive respiratory viral seasons (November to March) from 2009 to 2012 in the Children's University Hospital, Dublin (CUH), a tertiary level paediatric teaching hospital, and the associated neonatal outpatient department of the Rotunda Maternity Hospital, Dublin. Previously healthy Irish children of Caucasian ethnicity under the age of 18 months hospitalised for viral bronchiolitis were enrolled consecutively. Children with a history of chronic illness or immunodeficiency and children on immunosuppressants were excluded. Asymptomatic, age-matched controls, presenting for routine phlebotomy or minor day-case surgical procedures, were enrolled and were subject to similar exclusion criteria as cases. Written, informed consent was obtained from the parents/guardians of study participants. The Research Ethics Committees of the CUH and the Rotunda Hospital, Dublin approved the study.

\section{Study procedure}

All children with bronchiolitis were enrolled in CUH. Clinical and demographic information was collected by interviewing parents/guardians and examining medical records. Children were classified as having "severe" bronchiolitis if their condition was deemed by the attending paediatrician and intensivist to merit PICU admission for positive-pressure mechanical ventilator support and classified as "moderate" severity if the child was cared for on a standard inpatient ward. To further validate this classification, disease severity was also measured using a clinical score known as the Respiratory Distress Assessment Instrument (RDAI) [20]. The RDAI was calculated at the point of enrolment of each case by the same investigator (T.R.L.). Viral infection was confirmed in study participants using either direct fluorescence antibody staining of a nasopharyngeal aspirate and/or a multiplex respiratory viral real-time reverse transcription PCR of a nasopharyngeal swab [21]. Phlebotomy was performed once, shortly after enrolment, typically either on day 1 or 2 of admission. Clinical outcomes measured included length of hospital stay, the need for oxygen supplementation, PICU admission and mechanical ventilation.

\section{IL-15-related analysis}

Peripheral blood mononuclear cells (PBMCs) were extracted by density gradient centrifugation from an EDTA anticoagulated sample collected from each participant and quantitative real-time PCR (qPCR) for IL-15 mRNA levels was performed as previously described [22]. Serum was obtained from whole blood clotted at room temperature and IL-15 levels were measured by ELISA.

Flow cytometry

Lymphocyte phenotyping was performed on a CyAn-ADP flow cytometer instrument and analysed using Dako Summit software (Beckman Coulter, Pasadena, CA, USA). The relative frequency of lymphocyte 
subsets and surface expression of IL-2/15R $\beta$ were examined. Intracellular IL-15 production and expression of the IL-15-related antiapoptotic proteins $\mathrm{Bcl}-2$ and $\mathrm{Bcl}-\mathrm{x}_{\mathrm{L}}$ in different lymphocyte subset populations was also measured. Absolute numbers were extrapolated from a PBMC count undertaken on each sample using a Neubauer haemocytometer.

\section{NK cell microRNA (RNA) transcriptome and mRNA target gene analysis}

To determine whether the NK cell phenotype in infants with viral bronchiolitis was related to an altered pattern of miRNA gene expression, NK cells were isolated from PBMCs of six infants with RSV-positive bronchiolitis and six age-matched controls, and the transcriptional profile of small noncoding RNA compared using gene sequencing on a SOLiD 4 next-generation sequencing (NGS) platform (Life Technologies, Carlsbad, CA, USA) [23]. Bioinformatics analysis of the miRNA sequencing data identified miRNAs differentially expressed in infants with bronchiolitis. Statistical analysis was undertaken using open-source Bioconductor software DESeq2.0 (http://bioconductor.org/packages/release/bioc/html/DESeq2. html). This uses general linear regression modelling with a negative binomial distribution to identify differentially expressed genes. Raw p-values were adjusted for multiple comparisons using the BenjaminiHochberg method. Genes known to be targets of these miRNAs were identified by cross-referencing with miRWalk [24]. A number of these genes, known to be associated with control of the inflammatory response, were selected and their expression in NK cells compared between study groups using TaqMan (Life Technologies, Carlsbad, CA, USA) qPCR assays.

\section{Data analysis}

Descriptive analyses, means \pm SEM, medians (interquartile ranges (IQRs)) and frequency distributions were used to summarise the participants' baseline characteristics. As the data were, unless otherwise stated, nonparametric; study groups (controls, moderate, severe) were compared using either the Wilcoxon or Kruskal-Wallis rank sum test, with post-test comparison of moderate and severe groups using Dunn's multiple comparison test. Correlation was assessed using Spearman's rank correlation coefficient. A stepwise logistic regression model was applied to determine the independence of clinical features correlating to both IL-15 mRNA expression and serum IL-15 levels. $\mathrm{p}<0.05$ was considered statistically significant. Statistical analysis was performed using the JMP ${ }^{\circledast}$ (SAS, Cary, NC, USA) software package.

Further details of the methodology are available in the online supplementary material.

\section{Results}

\section{Clinical and demographic features}

Over the course of the study, 88 children with bronchiolitis and 43 controls were enrolled. Enrolment occurred across three respiratory viral seasons as demonstrated in figure 1. During the respiratory viral seasons of 2009/10 and 2010/11, 66 children with viral bronchiolitis and 30 controls were successfully enrolled. Their baseline characteristics are summarised on table 1. 61 of these children had RSV-positive bronchiolitis (92\%) including four children with co-infection. Virology results are summarised in online supplementary table E1. 22 of the 66 children had severe disease. A comparison of children with moderate and severe disease is presented in table 2. Samples collected from a second tranche of study participants (13 controls, 22 children with RSV-positive bronchiolitis) enrolled in 2011/12 were used for NK cell



FIGURE 1 Study enrolment. Analysis was performed on samples from 88 participants with bronchiolitis and 43 controls enrolled as illustrated. qPCR: quantitative real-time PCR; NK: natural killer; miRNA-Seq: microRNA sequencing. 


\begin{tabular}{|c|c|c|c|}
\hline & Controls & Viral bronchiolitis & $\mathrm{p}$-value \\
\hline Subjects $\mathbf{N}$ & 30 & 66 & \\
\hline Female $\mathrm{n}(\%)$ & $18(60)$ & $30(45.5)$ & $0.27^{\#}$ \\
\hline Birthweight kg & $3.4(3.0-3.9)$ & $3.4(2.8-3.8)$ & $0.70^{\pi}$ \\
\hline Age months & $5.8(0.7-12.4)$ & $2.2(1.2-6.7)$ & $0.43^{\pi}$ \\
\hline Gestational age at birth weeks/40 & $39.2(38-40.5)$ & $40(38-40)$ & $0.98^{\pi}$ \\
\hline Breastfed & $18 / 30(60)$ & $21 / 64(32.8)$ & $0.015^{\#}$ \\
\hline Number of siblings mean \pm SEM & $1.1 \pm 0.2$ & $1.3 \pm 0.1$ & $0.13^{\pi}$ \\
\hline Family history of atopy & $20 / 29(69.0)$ & $35 / 65(53.9)$ & $0.18^{\#}$ \\
\hline Parental smoking & $9 / 29(31.0)$ & $36 / 65(55.4)$ & $0.04^{\#}$ \\
\hline
\end{tabular}

Data are expressed as median values (25th-75th percentile interquartile range) or $\mathrm{n} / \mathrm{N}(\%)$, unless otherwise stated. " : calculated using Fisher's exact test; " ${ }^{\text {: }}$ calculated using the Wilcoxon rank sum test.

miRNA sequencing and NK cell mRNA PCR. Four of these 22 children had co-infection (three rhinovirus, two coronavirus). Their baseline characteristics are summarised in supplementary tables E2 and E3.

IL-15 mRNA expression in PBMCs and serum IL-15 levels

qPCR demonstrated a significant increase in IL-15 mRNA levels in PBMCs of children with bronchiolitis $(\mathrm{p}<0.0001)$. Participants with moderate severity bronchiolitis had statistically significantly higher expression than either controls $(\mathrm{p}<0.0001)$ or children with severe disease $(\mathrm{p}=0.01)$ (figure 2 and supplementary table E4). IL-15 protein levels as measured in the serum using ELISA (figure 2 and supplementary table E4) showed a statistically significant increment in levels from control to moderate $(p=0.0009)$ and from moderate to severe bronchiolitis $(\mathrm{p}=0.005)$.

Univariate analysis including potential confounders identified in tables 1 and 2 (breastfeeding, exposure to tobacco smoke (ETS) and prematurity) was performed (supplementary table E5), suggesting an association between ETS and IL-15 mRNA expression $(\mathrm{p}=0.02)$. However, this association was not maintained in a multivariate stepwise logistic regression model, confirming the independence of the association between IL-15 mRNA expression and both the occurrence of bronchiolitis (supplementary table E6; $\mathrm{p}=0.0003$ ) and its severity (supplementary table E7; $\mathrm{p}=0.009$ ).

TABLE 2 Comparison of participants with moderate and severe bronchiolitis

\begin{tabular}{|c|c|c|c|}
\hline & Moderate & Severe & p-value \\
\hline \multicolumn{3}{|l|}{ Demographics } & \\
\hline Age months & $2.6(1.2-8.5)$ & $1.7(1.2-2.2)$ & $0.08^{\#}$ \\
\hline Gestational age at birth weeks/40 & $40(39-41)$ & $37.5(34.1-40)$ & $0.0006^{\#}$ \\
\hline Premature $(<35 / 40$ weeks) at birth & $2 / 44(4.6)$ & $7 / 22(31.8)$ & $0.005^{\pi}$ \\
\hline Birthweight kg & $3.5(3.1-3.9)$ & $3.1(2.2-3.6)$ & $0.03^{\#}$ \\
\hline Parental smoking & $26 / 43(60)$ & $10 / 22(45)$ & $0.30^{\pi}$ \\
\hline Breastfed & $14 / 44$ (32) & $7 / 20(35)$ & $>0.99^{\natural 1}$ \\
\hline \multicolumn{4}{|l|}{ Viral characteristics } \\
\hline RSV infection & 40/44 (90.9) & $21 / 22(95.5)$ & $0.66^{\text {ก }}$ \\
\hline RSV qPCR $C_{T}$ value & $27.0(23.8-29.4)$ & $26.6(24.9-32.1)$ & $0.77^{\#}$ \\
\hline Non-RSV bronchiolitis n & $4(9.1)$ & $1(4.5)$ & $0.66^{\pi}$ \\
\hline Co-infection (two viruses) $n$ & $4(9.1)$ & $1(4.5)$ & $0.66^{9}$ \\
\hline \multicolumn{4}{|l|}{ Disease severity } \\
\hline RDAl score & $6(5-8)$ & $8.5(7-11.25)$ & $0.0022^{\#}$ \\
\hline Duration of hospital stay days & $3.8(2.6-6.7)$ & $11.1(8.2-15.3)$ & $<0.0001^{\#}$ \\
\hline Duration of oxygen therapy days & $2.3(0.8-4.9)$ & $8.3(3.6-12.5)$ & $<0.0001^{\#}$ \\
\hline \multicolumn{4}{|c|}{$\begin{array}{l}\text { Data are expressed as median values (25-75th percentile interquartile range) or n/N (\%), unless otherwise } \\
\text { stated. RSV infection denotes proportion of RSV-positive bronchiolitis among proven viral bronchiolitis cohort. } \\
\text { RSV: respiratory syncytial virus; qPCR: quantitative real-time PCR; RDAI: Respiratory Distress Assessment } \\
\text { Instrument. ": calculated using the Wilcoxon rank sum test; " }{ }^{\text {? }} \text { calculated using Fisher's exact test. }\end{array}$} \\
\hline
\end{tabular}





FIGURE 2 Interleukin (IL)-15 mRNA expression and serum IL-15 concentration in study participants. a) IL-15 mRNA copy number (normalised per 10 million copies of $\beta$-actin) in peripheral blood mononuclear cells of participants as measured by quantitative real-time PCR and b) serum IL-15 concentration as measured by ELISA. IL-15 mRNA copy number is highest in participants with moderate disease, whereas serum IL-15 levels are highest in those with severe disease.

Premature birth ( $<35$ weeks gestation) was found by univariate analysis to be significantly associated with reduced serum IL-15 levels (supplementary table E5; $\mathrm{p}=0.03$ ). However the statistical significance of this association was not maintained in multivariate analysis, which confirmed the independence of the association between serum IL-15 levels, the development of bronchiolitis (supplementary table E6; $\mathrm{p}=0.0002$ ) and severe disease (supplementary table E7; $\mathrm{p}=0.004$ ).

Chronological age at birth was also examined as a potential confounder. Neither the correlation between chronological age at birth and IL-15 mRNA expression (Spearman rho=0.185, p $>$ rho 0.1) nor serum IL-15 levels (Spearman $\mathrm{rho}=-0.01, \mathrm{p}>$ rho 0.94 ) were statistically significant.

\section{Lymphocyte phenotyping}

The concentrations of total PBMCs and lymphocytes were comparable between the three study groups (supplementary table E8). There was a statistically significant reduction in the relative frequency of NK cells $(p=0.008)$ in the peripheral blood of children with bronchiolitis as compared with the control group (supplementary figure E1). IL-2/15R $\beta$ was expressed predominantly on NK cells and significantly more frequently than on $\mathrm{T}$-cells ( $54 \%$ versus $2 \%$; $\mathrm{p}<0.0006)$.

\section{Intracellular IL-15 expression}

The cellular source of IL-15 in PBMCs was explored using intracellular cytokine staining, focusing initially on monocytes $\left(\mathrm{CD}^{-} \mathrm{CD} 14^{+}\right)$and later by examining the dendritic cell (DC) population, gating on the LIN1 ${ }^{-}$HLA-DR ${ }^{+}$population of PBMC. The relative distributions of myeloid $\left(\mathrm{CD} 11 \mathrm{c}^{+}\right)$, plasmacytoid $\left(\mathrm{CD} 123^{+}\right)$and double-negative $(\mathrm{DN})\left(\mathrm{CD} 11 \mathrm{c}^{-} \mathrm{CD} 123^{-}\right) \mathrm{DCs}$ are shown in supplementary table E9. Of note, children with bronchiolitis had a significantly higher percentage of DN DCs $(p=0.016)$ and a significantly lower percentage of plasmacytoid DCs $(p=0.001)$. The percentage of plasmacytoid DCs was significantly lower again in the severe group $(\mathrm{p}=0.017$ ). DN DCs were the predominant source of IL-15 (figure 3 and supplementary table E9) and IL-15 expression was seen in a significantly greater proportion of DN DCs in those with bronchiolitis $(\mathrm{p}=0.008)$. In children with viral bronchiolitis $(\mathrm{n}=6)$ and controls $(\mathrm{n}=2)$, intracellular expression of IL-15 in monocytes was almost undetectable (median $0.7 \%$ of cells, IQR $0.5-1.4 \%$ ).

\section{Expression of IL-15-related antiapoptotic markers}

Intracellular expression of $\mathrm{Bcl}-\mathrm{x}_{\mathrm{L}}$ was significantly greater in $\mathrm{CD}^{+} \mathrm{T}$-cells $(\mathrm{p}=0.007), \mathrm{CD}^{+} \mathrm{T}_{\text {-cells }}$ $(\mathrm{p}=0.03)$ and NK cells $(\mathrm{p}=0.003)$ of infants with bronchiolitis (supplementary table E10 and figure 4). Furthermore, the intensity of intracellular $\mathrm{Bcl}-\mathrm{x}_{\mathrm{L}}$ staining correlated with the serum concentration of IL-15, particularly in $\mathrm{NK}$ cells (Spearman rho=0.75, $\mathrm{p}=0.0051$ ). In children with bronchiolitis, the intracellular expression of Bcl-2 was significantly higher in $\mathrm{CD}^{+} \mathrm{T}$-cells $(\mathrm{p}=0.03)$, with a trend toward increased intracellular Bcl-2 in $\mathrm{CD}^{+} \mathrm{T}$-cells $(\mathrm{p}=0.08)$, but not in NK cells.

\section{Impact of RSV viral load on disease severity and immunological parameters}

Using the PCR cycle threshold $\left(\mathrm{C}_{\mathrm{T}}\right)$ value as a surrogate measure of RSV viral load, we did not detect any correlation (Spearman $\mathrm{rho}=0.03, \mathrm{p}=0.86$ ) with disease severity (RDAI). However, there was a slight trend towards lower NK cell percentage (Spearman $\mathrm{rho}=-0.33, \mathrm{p}=0.11$ ), higher serum IL-15 (Spearman rho $=-0.22, \mathrm{p}=0.18$ ) and IL-15 mRNA expression (Spearman rho $=-0.21, \mathrm{p}=0.2$ ) with lower $\mathrm{C}_{\mathrm{T}}$ values. 


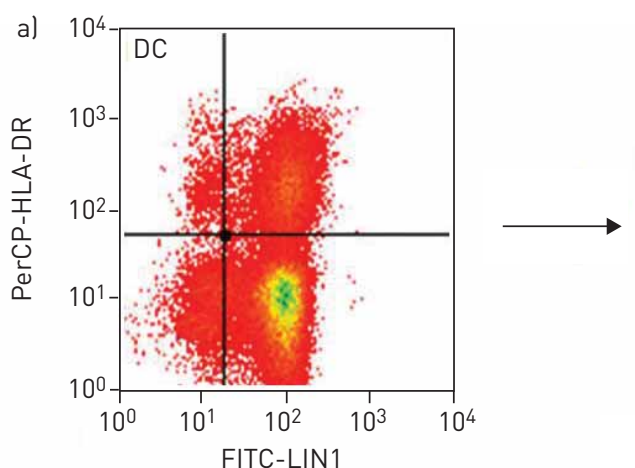

FITC-LIN1

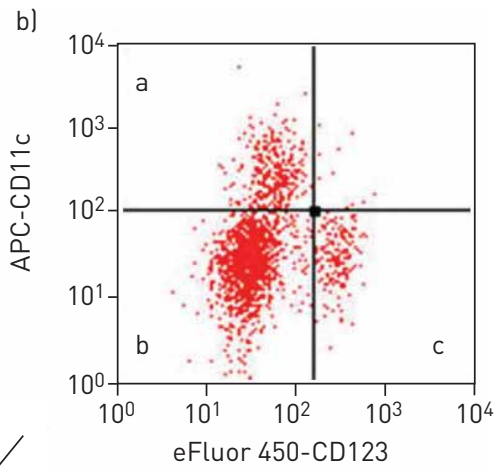

e)

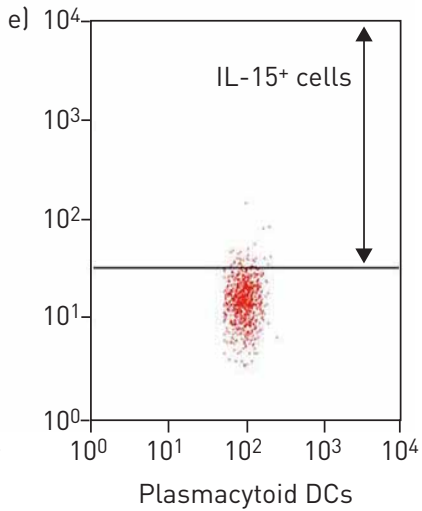

FIGURE 3 Relative intracellular expression of interleukin (IL)-15 in dendritic cell (DC) subsets. The DC population in peripheral blood mononuclear cells was identified a) as HLA-DR ${ }^{+} \mathrm{IN1}^{-}$(labelled DC) and subsets identified b) using cell surface staining for plasmacytoid (CD11 $c^{-}$CD $\left.123^{+}\right)$and myeloid (CD11 $\left.c^{+} C D 123^{-}\right)$DC markers. IL-15 was found intracellularly in a significantly higher proportion of c) DN (CD11c $\left.{ }^{-} C D 123^{-}\right)$DCs compared with d) myeloid DCs and e) plasmacytoid DCs among children with viral bronchiolitis. DN: double-negative; PerCP: peridinin chlorophyll protein; FITC: fluorescein isothiocyanate; APC: allophycocyanin.

\section{NK cell miRNA sequencing and relative quantification of putative MRNA targets by PCR}

NK cells (93\% purity from extracted PBMC population) isolated from six infants with RSV bronchiolitis and six age-matched controls exhibited an array of differentially expressed miRNAs and other small noncoding RNAs as listed in table 3. Putative target genes for differentially expressed miRNAs and their relative expression in NK cells are listed in table 4.

There was a statistically significant intergroup variation in 12 of the 19 selected mRNAs targets. Seven target mRNAs, including three genes on the IL-15 pathway (JAK3, STAT5A and NFKB1), were differentially expressed in those with severe bronchiolitis compared with those with moderate severity bronchiolitis.

\section{Discussion}

We demonstrate, for the first time to the best of our knowledge, an association between disease severity in children hospitalised with viral bronchiolitis and 1) IL-15 mRNA expression in PBMCs and 2) IL-15 concentration in serum. We identified the source of IL-15 in PBMCs as a subpopulation of DCs that are expanded in viral bronchiolitis, and also report a correlation between IL-15 levels and intracellular expression of antiapoptotic proteins $\mathrm{Bcl}-2$ and $\mathrm{Bcl}-\mathrm{x}_{\mathrm{L}}$ in lymphocytes of children with bronchiolitis. We identified a distinct pattern of NK cell miRNA expression in bronchiolitis and differential expression of a selection of their putative mRNA targets, among them genes for signalling molecules in the IL-15 pathway.

Children with viral bronchiolitis in our cohort demonstrated upregulation of IL-15 mRNA expression in PBMCs and increased serum levels of IL-15. This is consistent with an appropriate innate immune response to a viral infection, as it promotes activation and recruitment of NK cells to the lungs. However, while there is an increase in the serum concentration of IL-15 with increasing disease severity, in contrast children with severe bronchiolitis in our study did not demonstrate upregulation of PBMC IL-15 mRNA expression. There may be a number of explanations for the lack of correlation between serum IL-15 levels and PBMC IL-15 mRNA expression in severe bronchiolitis. First, serum IL-15 levels reflect production by 

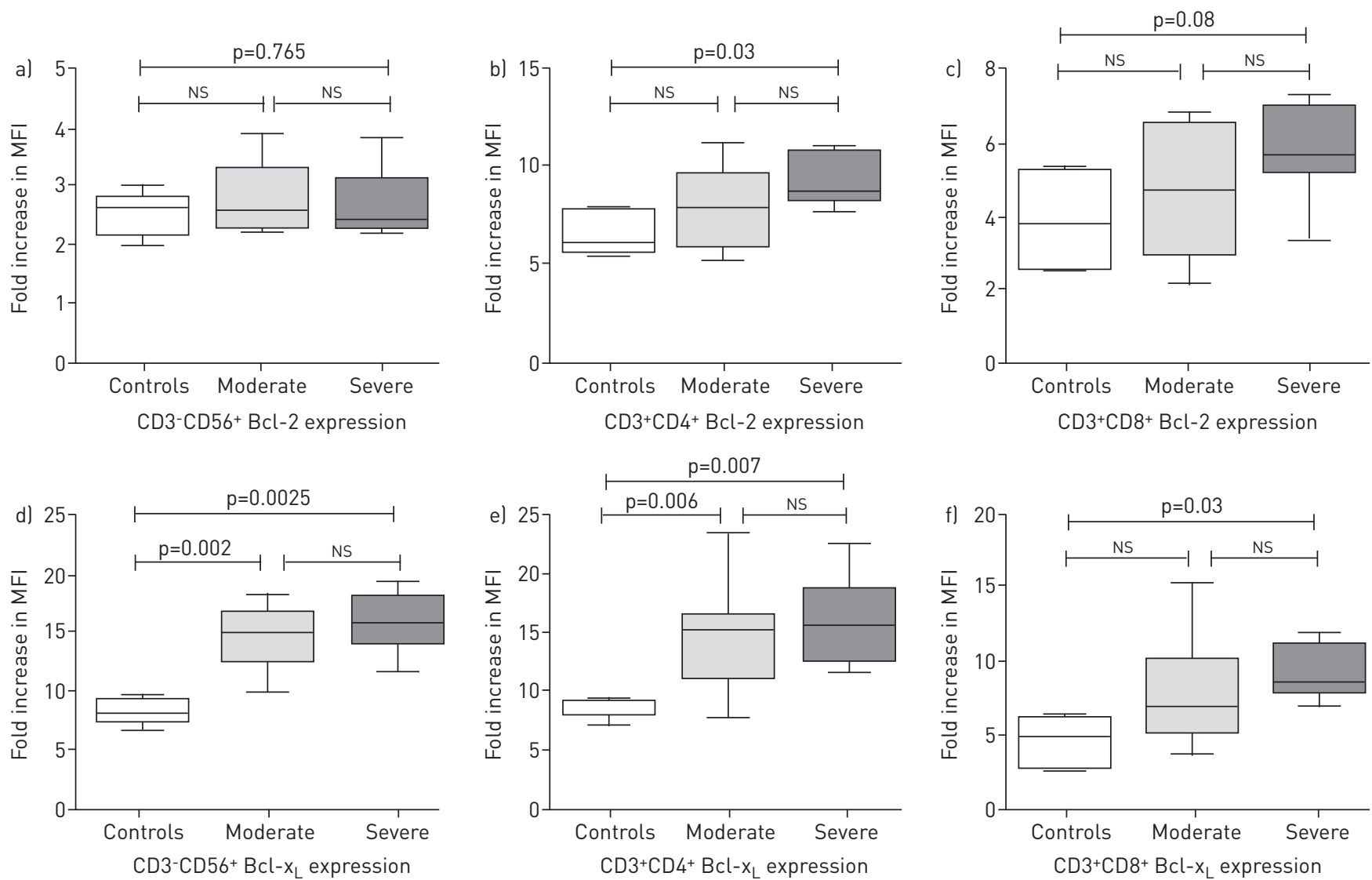

FIGURE 4 Intracellular expression of antiapoptotic proteins by lymphocyte subset in study participants. Bcl-2 expression in a) NK cells did not differ between controls and children with bronchiolitis, unlike b) $C D 4^{+} T$-cells and c) $C D 8^{+} \mathrm{T}$-cells. Bcl- $\mathrm{x}_{\mathrm{L}}$ expression was increased in participants with bronchiolitis in d) NK cells, e) $\mathrm{CD}^{+} \mathrm{T}$-cells and f) $\mathrm{CD} 8^{+} \mathrm{T}$-cells. MFI: mean fluorescence intensity; NK: natural killer.

all cells, whereas IL-15 mRNA expression, as measured in our experiment, reflects only mRNA expression in the extracted PBMC. The difference may also reflect a homeostatic measure. IL-15 expression is tightly regulated at the mRNA level, as unrestrained NK cell activation may have harmful effects and has been shown to lead to pulmonary fibrosis in the mouse model [25]. In addition, IL-15 produced by antigen-presenting cells (APCs) such as DCs and mononuclear phagocytes within the PBMC compartment is trans-presented to the target cell together with IL-15R $\alpha$, allowing the target cells to respond at lower physiological levels of IL-15 [26]. Therefore, PBMC IL-15 mRNA expression may be more carefully regulated than in nonimmune cells.

Alternatively, failure to upregulate IL-15 mRNA expression may represent evidence of an "immunoparesis" among those children with severe bronchiolitis, analogous to that demonstrated by our research group in sepsis and pneumonia in other patient cohorts [27]. Immunoparesis in this context may be genetically mediated and may, at least in part, account for the inherited susceptibility to severe bronchiolitis seen among some children [28]. Support for this hypothesis comes from genetic association studies undertaken and published by our colleagues in Utrecht, The Netherlands [6]. In one of the biggest studies of its kind, JANSSEN et al. [6] showed an overall association of innate immunity genes with genetic susceptibility to RSV infection in a candidate gene study of 480 hospitalised children and $>1000$ controls. Among other associated loci, they showed a nominal association with alleles of the IL15 gene variant rs2254514 (OR 1.22, 95\% CI 1.02-1.46; $\mathrm{p}=0.026$ ) and hospitalisation for RSV. Interestingly, rs2254514 is a proxy for another IL15 SNP rs6837991 (linkage disequilibrium; $\mathrm{r}^{2}=0.87$ ), which has a RegulomeDB (http://www. regulomedb.org/) score of $1 \mathrm{f}$ (i.e. it is likely to affect IL15 gene expression). Thus, while the association is weak, due to the limitations of power of the original study, there is inferential evidence of a genetic association with RSV susceptibility and IL15 expression [29]. Finally, absence of PBMC IL-15 upregulation in severe bronchiolitis may reflect a viral pathogenic effect. RSV, for example, has been shown to infect human monocyte-derived DCs in vitro, and impair their ability to subsequently activate and proliferate T-cells [30]. A similar phenomenon might explain the findings in this study. 
TABLE 3 Differentially expressed small noncoding RNA in enriched natural killer cells from infants with respiratory syncytial virus-positive bronchiolitis compared with healthy age-matched controls

\begin{tabular}{|c|c|c|c|c|}
\hline Gene & Transcript type & Base mean & $\log _{2}$ fold increase & Adjusted p-value \\
\hline \multicolumn{5}{|l|}{ Upregulated } \\
\hline SNORD82 & snoRNA & 117.497 & 1.87 & 0.022 \\
\hline MIR370 & miRNA & 132.383 & 3.785 & 0.041 \\
\hline SCARNA6 & snoRNA & 134.66 & 1.589 & 0.022 \\
\hline MIR3074 & miRNA & 160.91 & 1.083 & 0.009 \\
\hline MIR379 & miRNA & 177.244 & 3.233 & 0.022 \\
\hline SNORD85 & snoRNA & 247.918 & 1.332 & 0.014 \\
\hline SCARNA18 & snoRNA & 289.76 & 1.644 & 0.022 \\
\hline MIR222 & miRNA & 485.265 & 2.288 & 0.014 \\
\hline AC078899.5 & miRNA & 691.042 & 1.41 & 0.01 \\
\hline SCARNA5 & snoRNA & 1265.42 & 1.473 & 0.014 \\
\hline MIR101-1 & miRNA & 1378.251 & 1.335 & 0.022 \\
\hline MIR30D & miRNA & 1427.109 & 1.041 & 0.027 \\
\hline U8 & snoRNA & 1445.441 & 1.349 & 0.004 \\
\hline snoU2-30 & snoRNA & 1538.635 & 1.735 & 0.017 \\
\hline SNORD73A & snoRNA & 3053.163 & 1.384 & 0.022 \\
\hline MIR873 & miRNA & 6541.255 & 1.113 & 0.049 \\
\hline SNORD51 & snoRNA & 7180.6 & 1.328 & 0.029 \\
\hline SNORD74 & snoRNA & 7471.941 & 1.786 & 0.017 \\
\hline RNY4P10 & misc_RNA & 13473.821 & 2.792 & 0.022 \\
\hline MIR103A2 & miR̄NA & 63043.74 & 1.264 & 0.013 \\
\hline MIR221 & miRNA & 156248.869 & 2.233 & 0.045 \\
\hline \multicolumn{5}{|l|}{ Downregulated } \\
\hline RN7SL610P & misc_RNA & 340.915 & -1.865 & 0.014 \\
\hline RPPH1 & misc_RNA & 794.029 & -2.07 & 0.022 \\
\hline$Y \_R N A$ & misc_RNA & 2615.626 & -1.219 & 0.025 \\
\hline$\overline{R N 7 S L 364 P}$ & misc_RNA & 101.251 & -1.716 & 0.025 \\
\hline LA16c-36OH6.3 & Antisense RNA & 7897.37 & -1.635 & 0.027 \\
\hline$R P 11-1129 / 3.1$ & Sense overlapping & 150.593 & -2.302 & 0.049 \\
\hline MIR199B & miRNA & 1706.133 & -2.165 & 0.049 \\
\hline
\end{tabular}

snoRNA: small noncoding RNA; miRNA: microRNA; misc_RNA: miscellaneous RNA.

IL-15 is particularly important for NK cell homeostasis and activation [31]. We hypothesised that children with severe bronchiolitis had a defect in the APC-IL-15-NK cell axis that prevented the development of a competent innate response to respiratory viral infection. This might account for the paucity of NK cells in lung tissue seen at autopsy studies of children who died from severe viral bronchiolitis [32]. Lymphocyte phenotyping by flow cytometry revealed a significant drop in the relative percentage of NK cells seen in the peripheral blood among children with bronchiolitis. While noteworthy, a similar finding has been reported previously [33]. Furthermore, LARRANaGa et al. [34] reported changes in NK cell subsets, in particular unregulated $\mathrm{CD} 94^{+}$activated NK cells, correlating with disease severity in RSV bronchiolitis. These changes in NK cell and subset distribution may represent sequestration of activated NK cells to the site of infection rather than apoptosis. In support of this conjecture, we found increased expression of the IL-15-related antiapoptotic marker $\mathrm{Bcl}-\mathrm{x}_{\mathrm{L}}$ in lymphocytes (including $\mathrm{NK}$ cells) in children with bronchiolitis. In contrast, Bcl-2 expression was not upregulated in NK cells of children with bronchiolitis. The significance of this finding needs to be explored further in a larger cohort.

We identified a subpopulation of DCs as the source of IL-15 in PBMCs of infants and found this population to be expanded among children with bronchiolitis. This argues against cellular depletion as an explanation for decreased IL-15 mRNA in severe bronchiolitis. DCs are known to be a cellular source of IL-15, but the change in DC subset distribution and IL-15 production demonstrated in children with bronchiolitis has not been reported previously [35].

Results from qPCR, ELISA and flow cytometry experiments led us to screen for differential expression of miRNA in NK cells of infants with RSV bronchiolitis, miRNA now being increasingly recognised as central to NK cell function [36]. We observed that NK cells of infants with RSV exhibited a distinct pattern of miRNA expression. Even with our limited sample size ( $\mathrm{n}=6$ in each group), statistically significant differences were detectable using current analytic methods [37]. 
TABLE 4 Relative quantification of mRNA targets of microRNA genes with altered expression by study group

\begin{tabular}{lccccc} 
& Control & Moderate & Severe & p-value & p-value $^{\text {I }}$ \\
\hline Subjects n & 13 & 14 & 8 & & \\
AKT1 & $1.1(1.0-1.2)$ & $1.3(1.2-1.5)$ & $1.3(1.0-1.8)$ & 0.01 & NS \\
AKT2 & $1.7(1.4-1.9)$ & $1.9(1.7-2.1)$ & $1.7(1.5-2.5)$ & 0.18 & NS \\
BAX & $1.2(0.9-1.4)$ & $1.7(1.4-1.9)$ & $1.7(1.5-2.0)$ & 0.0029 & NS \\
CXCR4 & $1.2(0.5-2.4)$ & $1.6(1.0-2.0)$ & $1.4(0.7-2.9)$ & 0.78 & NS \\
FAS & $0.2(0.2-0.3)$ & $0.5(0.4-1.0)$ & $0.7(0.6-1.3)$ & 0.0001 & 0.0478 \\
FLT3 & $1.1(0.5-1.5)$ & $1.3(0.5-1.9)$ & $3.4(2.2-4.2)$ & 0.0066 & 0.0041 \\
FOX03 & $0.5(0.4-1.2)$ & $0.7(0.6-0.9)$ & $0.6(0.5-2.1)$ & 0.86 & NS \\
GADPH & $1.0(0.9-1.1)$ & $1.3(1.0-1.6)$ & $3.3(1.8-5.0)$ & 0.0008 & 0.0041 \\
IRAK1 & $1.1(0.9-1.1)$ & $1.4(1.2-1.6)$ & $1.8(1.4-2.2)$ & 0.0013 & NS \\
JAK3 & $0.6(0.5-0.8)$ & $0.9(0.8-1.0)$ & $1.9(1.3-2.9)$ & $<0.0001$ & 0.0021 \\
MAPK1 & $0.9(0.9-1.0)$ & $1.1(1.0-1.3)$ & $1.3(1.1-1.7)$ & 0.0037 & NS \\
MAPK8 & $1.2(1.2-1.3)$ & $1.2(1.1-1.6)$ & $1.3(1.0-1.9)$ & 0.75 & NS \\
NFATC2 & $1.4(1.3-1.5)$ & $1.7(1.3-2.0)$ & $1.3(0.4-2.0)$ & 0.14 & NS \\
NFKB1 & $0.7(0.6-0.8)$ & $1.0(0.9-1.1)$ & $1.3(1.0-1.7)$ & $<0.0001$ & 0.0203 \\
STAT1 & $0.4(0.4-0.7)$ & $0.8(0.6-1.6)$ & $1.3(0.6-3.1)$ & 0.007 & NS \\
STAT5A & $1.0(0.7-1.2)$ & $1.2(1.0-1.5)$ & $1.9(1.3-2.6)$ & 0.0048 & 0.0290 \\
TGFB1 & $1.3(1.1-1.4)$ & $1.3(1.2-1.6)$ & $1.3(1.1-1.7)$ & 0.63 & NS \\
TGFB2 & $1.0(0.6-2.9)$ & $1.1(0.7-2.1)$ & $4.9(1.3-8.2)$ & 0.17 & NS \\
TNFRSF10A & $1.8(1.2-2.0)$ & $1.9(1.1-2.6)$ & $3.9(2.3-5.4)$ & 0.0155 & 0.0203 \\
\hline
\end{tabular}

Relative quantity values are expressed as median (interquartile range), unless otherwise stated. Ns: nonsignificant.

\#: calculated using the Kruskal-Wallis rank sum test to compare all three groups; ": calculated using Dunn's multiple comparison test to compare moderate versus severe bronchiolitis.

Bioinformatics analysis identified putative target genes for these deregulated miRNAs. Differential expression of a selection of these target genes was confirmed in NK cells of infants with moderate and severe bronchiolitis. These differentially expressed target genes included genes regulating cell activation and expansion; notably STAT5A, JAK3 and NFKB1, which code for proteins on the IL- $15 / \gamma_{c}$ signalling pathway, reinforcing the hypothesis that IL-15 is linked to disease severity in RSV bronchiolitis [38].

The patterns of miRNA expression that characterised the NK phenotype of infants with bronchiolitis are distinct from those reported in prior in vitro studies where NK cells were activated by $\gamma_{c}$ cytokines IL-2 and IL-15 [23, 39]. However, in vitro experiments of NK cell activation may not reflect the complexity of a clinical disease process. In this regard, our study adds to the available NK cell literature, providing a novel insight into factors that regulate NK cell activation in infants with viral infection.

Our study has several limitations. Being a single-centre study, our capacity to recruit large numbers was limited, restricting the power of our study. We attempted to mitigate this risk by collaborating with colleagues in The Netherlands, who having access to a much larger cohort of patients, report a link between IL-15 single nucleotide polymorphisms (SNPs) and disease severity. There are limitations to this approach, as patient cohorts are distinct and recruited by separate investigators. However, we consider that the large size of the Dutch cohort adds considerable strength to the conclusions from our smaller study of IL-15 gene expression. Some potential confounders, notably gestational age at birth, breastfeeding rate and ETS, distinguish those in the moderate and severe groups. However, the link between disease and IL-15 was maintained when these confounders were accounted for in multivariate analysis. Small volumes of blood were obtained from those patients recruited, which meant that not all tests could be performed on each patient sample. The technical challenges associated with extracting sufficient miRNA from small volumes of blood containing relatively few NK cells introduced an element of bias into the sequencing data, as those with the most severe disease had the fewest NK cells, precluding their analysis. This precluded an exploration of the relationship between $\mathrm{NK}$ cell $\mathrm{miR}$, disease progression and resolution. The results of miRNA transcriptomal screening involving multiple comparisons and performed in relatively small numbers of patients are inferential in nature. To validate these inferential transcriptomal findings, we confirmed differential expression of miRNA target genes in NK cells. As with all biomarker studies, external validation of these findings in cohorts from different patient populations using different assays and platforms is needed.

Despite these limitations, data garnered from SNP analysis, qPCR, ELISA, flow cytometry and NGS all suggest an association between IL-15 and disease severity in children hospitalised with viral bronchiolitis. This may serve to prompt research into novel RSV vaccination and treatment strategies. Further research is 
needed to examine the relative impact of the other $\gamma_{c}$ cytokines on disease severity in RSV bronchiolitis, as well as the specific contribution of other innate cells to the clinical phenotype.

\section{Acknowledgements}

The authors wish to acknowledge the goodwill of parents of all study participants, of the staff of both the CUH and the Rotunda Hospital, and of the many fundraisers for the Children's Fund for Health, without whom this study could not have been completed.

\section{References}

1 Simoes EAF. RSV disease in the pediatric population: epidemiology, seasonal variability, and long-term outcomes. Manag Care 2008; 17: 11 Suppl. 12, 3-6.

2 Cody Meissner H. Bronchiolitis. In: Long S, Pickering L, Prober C, eds. Principles and practice of pediatric infectious diseases. 3rd edn. Amsterdam, Elsevier, 2008; pp. 241-245.

3 Smyth RL, Openshaw PJ. Bronchiolitis. Lancet 2006; 368: 312-322.

4 Welliver RC. Review of epidemiology and clinical risk factors for severe respiratory syncytial virus (RSV) infection. J Pediatr 2003; 143: 5 Suppl., S112-S117.

5 Purcell K, Fergie J. Driscoll Children's Hospital respiratory syncytial virus database: risk factors, treatment and hospital course in 3308 infants and young children, 1991 to 2002. Pediatr Infect Dis J 2004; 23: 418-423.

6 Janssen R, Bont L, Siezen CL, et al. Genetic susceptibility to respiratory syncytial virus bronchiolitis is predominantly associated with innate immune genes. J Infect Dis 2007; 196: 826-834.

7 Mella C, Suarez-Arrabal MC, Lopez S, et al. Innate immune dysfunction is associated with enhanced disease severity in infants with severe respiratory syncytial virus bronchiolitis. J Infect Dis 2013; 207: 564-573.

8 Lodoen MB, Lanier LL. Natural killer cells as an initial defense against pathogens. Curr Opin Immunol 2006; 18: $391-398$

9 Stein-Streilein J, Bennett M, Mann D, et al. Natural killer cells in mouse lung: surface phenotype, target preference, and response to local influenza virus infection. J Immunol 1983; 131: 2699-2704.

10 Stein-Streilein J, Guffee J, Fan W. Locally and systemically derived natural killer cells participate in defense against intranasally inoculated influenza virus. Reg Immunol 1988; 1: 100-105.

11 Ennis FA, Meager A, Beare AS, et al. Interferon induction and increased natural killer-cell activity in influenza infections in man. Lancet 1981; 2: 891-893.

12 Kos FJ, Engleman EG. Role of natural killer cells in the generation of influenza virus-specific cytotoxic T cells. Cell Immunol 1996; 173: 1-6.

13 Martin-Fontecha A, Thomsen LL, Brett S, et al. Induced recruitment of NK cells to lymph nodes provides IFN-gamma for $\mathrm{T}_{\mathrm{H}} 1$ priming. Nat Immunol 2004; 5: 1260-1265.

14 Welliver TP, Reed JL, Welliver RC Sr. Respiratory syncytial virus and influenza virus infections: observations from tissues of fatal infant cases. Pediatr Infect Dis J 2008; 27: 10 Suppl., S92-S96.

15 Waldmann TA. The biology of interleukin-2 and interleukin-15: implications for cancer therapy and vaccine design. Nat Rev Immunol 2006; 6: 595-601.

16 Huntington ND. The unconventional expression of IL-15 and its role in NK cell homeostasis. Immunol Cell Biol 2014; 92: 210-213.

17 Ferlazzo G, Pack M, Thomas D, et al. Distinct roles of IL-12 and IL-15 in human natural killer cell activation by dendritic cells from secondary lymphoid organs. Proc Natl Acad Sci U S A 2004; 101: 16606-16611.

18 Grzeoek E, Koltan S, Debski R, et al. Concentrations of IL-15, IL-18, IFN-gamma and activity of CD4 ${ }^{+}$, CD8 ${ }^{+}$and NK cells at admission in children with viral bronchiolitis. Exp Ther Med 2010; 1: 873-877.

19 Leahy T, Slattery D, Doherty D, et al. Innate lymphocytes frequency and gene expression of innate cytokines predict disease severity in viral bronchiolitis. Presented at ESPID annual meeting, The Hague, 2011 ; abstract O033. http://espid.kenes.com/Documents/ESPID2011\%20ABSTRACTS.pdf Date last accessed: July 10, 2014.

20 Klassen TP, Sutcliffe T, Watters LK, et al. Dexamethasone in salbutamol-treated inpatients with acute bronchiolitis: a randomized, controlled trial. J Pediatr 1997; 130: 191-196.

21 Gunson RN, Carman WF. During the summer 2009 outbreak of "swine flu" in Scotland what respiratory pathogens were diagnosed as H1N1/2009? BMC Infect Dis 2011; 11: 192.

22 Stordeur P, Poulin LF, Craciun L, et al. Cytokine mRNA quantification by real-time PCR. J Immunol Methods 2002; 259: 55-64.

23 Fehniger TA, Wylie T, Germino E, et al. Next-generation sequencing identifies the natural killer cell microRNA transcriptome. Genome Res 2010; 20: 1590-1604.

24 Dweep H, Sticht C, Pandey P, et al. miRWalk - database: prediction of possible miRNA binding sites by "walking" the genes of three genomes. J Biomed Inform 2011; 44: 839-847.

25 Okamoto M, Kato S, Oizumi K, et al. Interleukin 18 (IL-18) in synergy with IL-2 induces lethal lung injury in mice: a potential role for cytokines, chemokines, and natural killer cells in the pathogenesis of interstitial pneumonia. Blood 2002; 99: 1289-1298.

26 Stonier SW, Schluns KS. Trans-presentation: a novel mechanism regulating IL-15 delivery and responses. Immunol Lett 2010; 127: 85-92.

27 White M, Mahon V, Grealy R, et al. Post-operative infection and sepsis in humans is associated with deficient gene expression of $\gamma_{c}$ cytokines and their apoptosis mediators. Crit Care 2011; 15: R158.

28 Thomsen SF, Stensballe LG, Skytthe A, et al. Increased concordance of severe respiratory syncytial virus infection in identical twins. Pediatrics 2008; 121: 493-496.

29 Bhoj VG, Sun Q, Bhoj EJ, et al. MAVS and MyD88 are essential for innate immunity but not cytotoxic T lymphocyte response against respiratory syncytial virus. Proc Natl Acad Sci U S A 2008; 105: 14046-14051.

30 de Graaff PM, de Jong EC, van Capel TM, et al. Respiratory syncytial virus infection of monocyte-derived dendritic cells decreases their capacity to activate CD4 T cells. J Immunol 2005; 175: 5904-5911.

31 Cooper MA, Bush JE, Fehniger TA, et al. In vivo evidence for a dependence on interleukin 15 for survival of natural killer cells. Blood 2002; 100: 3633-3638. 
Welliver TP, Garofalo RP, Hosakote Y, et al. Severe human lower respiratory tract illness caused by respiratory syncytial virus and influenza virus is characterized by the absence of pulmonary cytotoxic lymphocyte responses. J Infect Dis 2007; 195: 1126-1136.

33 De Weerd W, Twilhaar WN, Kimpen JL. T cell subset analysis in peripheral blood of children with RSV bronchiolitis. Scand J Infect Dis 1998; 30: 77-80.

34 Larranaga CL, Ampuero SL, Luchsinger VF, et al. Impaired immune response in severe human lower tract respiratory infection by respiratory syncytial virus. Pediatr Infect Dis J 2009; 28: 867-873.

35 Jonuleit $\mathrm{H}$, Wiedemann $\mathrm{K}$, Muller G, et al. Induction of IL-15 messenger RNA and protein in human blood-derived dendritic cells. J Immunol 1997; 158: 2610-2615.

36 Sullivan RP, Leong JW, Fehniger TA. MicroRNA regulation of natural killer cells. Front Immunol 2013; 4: 44.

37 Rapaport F, Khanin R, Liang Y, et al. Comprehensive evaluation of differential gene expression analysis methods for RNA-seq data. Genome Biol 2013; 14: R95.

38 Johnston JA, Bacon CM, Finbloom DS, et al. Tyrosine phosphorylation and activation of STAT5, STAT3, and Janus kinases by interleukins 2 and 15. Proc Natl Acad Sci U S A 1995; 92: 8705-8709.

39 Liu X, Wang Y, Sun Q, et al. Identification of microRNA transcriptome involved in human natural killer cell activation. Immunol Lett 2012; 143: 208-217. 